\title{
Influence of acrylic adhesive viscosity and surface roughness on the properties of adhesive joint
}

\author{
Eva Paz ${ }^{1}$, Professor Julián José Narbón ${ }^{2}$, Dr J. Abenojar ${ }^{3}$, Dr Mar Cledera ${ }^{1}$, Dr. Juan \\ Carlos del Real-Romero \\ ${ }^{1}$ Institute for Research in Technology (IIT)/Mechanical Engineering Dept., Universidad \\ Pontificia Comillas. Madrid, Spain, \\ ${ }^{2}$ Escuela Técnica Superior de Ingeniería y Diseño Industrial, Universidad Politécnica de \\ Madrid, Spain, \\ ${ }^{3}$ Materials Performance Group, Materials Science and Engineering Dept, Universidad \\ Carlos III de Madrid, Spain \\ Corresponding author: Eva Paz, Institute for Research in Technology (IIT), Universidad \\ Pontificia Comillas, c/ Alberto Aguilera 23, 28015 Madrid, Spain. \\ Email: eva.paz@iit.upcomillas.es
}

Received: 01 December 2014; revised: 11 May 2015; accepted: 11 May 2015

\begin{abstract}
In adhesion, the wetting process depends on three fundamental factors: the surface topography of the adherend, the viscosity of the adhesive, and the surface energy of both. The aim of this paper is to study the influence of viscosity and surface roughness on the wetting and their effect on the bond strength. For this purpose, an acrylic adhesive with different viscosities was synthesized and some properties, such as viscosity and surface tension, were studied before adhesive curing took place. Furthermore, the contact angle and the lap-shear strength were analyzed using aluminum adherends with two different roughnesses. Scanning electron microscopy was used to determine the effect of the viscosity and the roughness on the joint interface. The results showed that the adhesive exhibits an optimal value of viscosity. Below this value, at low viscosities, the low neoprene content produces poor bond strength due to the reduced toughness of the
\end{abstract}


adhesive. Additionally, it also produces a high shrinkage during curing, which leads to the apparition of residual stresses that weakens the interfacial strength. However, once the optimum value, an increase in the viscosity produces a negative effect on the joint strength as a result of an important decrease in the wettability.

KEYWORDS: Surface roughness, Wetting, Rheology, Lap-shear, Structural acrylics

\section{INTRODUCTION}

Adhesives exhibit very different rheological properties before and after curing. The rheology of adhesives is a very important issue concerning adhesives during dispensing, mainly when automatic dispensing systems are used [1]. In addition, the rheological properties of adhesives define characteristics such as: processability, application, wettability, etc. [2]

Prior to curing, adhesives should act as fluids, capable of being transported to the adherend surface and should copy the surface morphology via sufficient wetting of the surface. During the curing process, the adhesive increases its viscosity until it is fully cured. At this moment, the adhesive reaches the adequate mechanical properties for the joint requirements.

Since the phenomenon of adhesion is a surface phenomenon, the surface micro-geometry has a great influence on the strength of the adhesive bond $[3,4]$. It has been demonstrated that a surface treatment substantially improves the strength and durability of the joint [ 5 , 
6]. However the exact relationship between strength, durability and surface roughness is difficult to predict because it depends on many factors (e.g. wetting, surface treatment, etc.) and can vary as a result of the adhesive type. Penetration of the adhesive into the structure created by the surface treatment is an important parameter to achieve durable bonds [7]. Other authors argue that a rougher surface produces an increase of the actual surface area and, therefore, increases the potential for multiple bonds between the adhesive and the substrate [8]. In any case, both effects have positive influence on the adhesion. However, if the roughness is too high, the level of strength can decrease. Some authors have shown that there is a critical value of roughness for which the joint exhibits the maximum strength $[4,9]$. This critical value depends on the surface treatment and the properties of the adherend and the adhesive, which include wettability and viscosity. From this critical value of roughness, there appears to be problems associated with trapped air bubbles and reduced wettability of the adhesive, which may cause a reduction in joint strength [10].

In order to achieve good adhesion, it is essential to conduct a satisfactory wetting of the adherend by the adhesive [11]. This wetting is strongly influenced by surface roughness $[12,13]$. Roughness may affect the penetration of the adhesive into the pores and topographical features of the adherend surface [14].

Wettability can be studied by contact angle measurements, among other techniques. The contact angle may be defined as the angle formed between the adherend and a drop of adhesive deposited when the system reaches equilibrium. 
The contact angle on a smooth and a rough surface can be calculated by the roughness factor, according to Wenzel's equation [15]:

$\cos \theta_{\text {rough }}=r \cdot \cos \theta_{\text {smooth }}$

Where $\theta_{\text {rough }}$ the rugged contact angle on the rough surface, $\theta_{\text {smooth }}$ the smooth contact angle balanced on the smooth surface and roughness factor characteristic of the roughened surface (actual surface/ nominal surface).

Some studies [16] have shown that the surface treatment may change the surface, not only on a morphological level, but also on a chemical level, which could modify the surface energy of the adherend and cause chemically heterogeneous surfaces and alter the wetting at the same time. In addition, the speed at which the droplet deposited on the surface of the adherend reaches equilibrium depends on various factors, such as the capillary forces, whose origin is the surface tension, $\gamma$, and viscosity $\eta$.

Therefore, the viscosity of the adhesive can also influence the penetration into the voids and surface roughness in terms of the speed with which the wetting occurs. This effect could modify the equilibrium predicted by the surface tension of the adhesive. This behavior may affect the wetting process and consequently the adhesion strength [11].

The aim of this study was to investigate the effect of adherend surface roughness and adhesive viscosity on wettability. For that purpose, an acrylic adhesive with different contents of neoprene was synthesized. In order to evaluate their mechanical behavior, the lap-shear strength was also studied using an aluminum alloy as an adherend. 


\section{EXPERIMENTAL}

\subsection{Materials}

In this study an acrylic adhesive was synthesized in the laboratory, based on some formulations used by other authors [17]. The adhesive composition is shown in Table 1. A commercial activator (a condensation product of an amine and an aldehyde), Loctite 7388 (Henkel Iberica, Barcelona, Spain) was used as cure activator.

The different viscosity values for the adhesives used in this study were obtained by modifying the concentration of neoprene in a range between $8 \%$ and $16 \%$, in respect of the total mass of monomers.

During the manufacturing process of the adhesive, neoprene was dissolved in methyl methacrylate by magnetic stirrer agitation at room temperature over a period of 24 hours. The cure initiator was added just before the adhesive application. The activator was applied directly onto the substrate.

The adherend used was an aluminum alloy EN AW 6082-T6 treated by mechanical abrasion through sandpaper to achieve two different levels of roughness, $\mathrm{Ra}=0.2 \mu \mathrm{m}$ and $\mathrm{Ra}=2 \mu \mathrm{m}$. The sandpaper grit used was P600 in the first case and P60 in the second case. The roughness was measured with a contact profilometer Type SJ-201 P (Mitutoyo, Neuss, Germany).

\subsection{Determination Of Viscosity}


The viscosity of the adhesive in relation to the content of neoprene was studied with a rotational viscosimeter Fungilab Smart Serie (Fungilab, Barcelona, Spain) according to UNE 12092:2002. The measurements were made with a R2 spindle of stainless steel at a rotation speed of $1.5 \mathrm{rpm}$. Five adhesive formulations with different contents of neoprene $(8,10,12,14$, and $16 \mathrm{wt} \%)$ were tested.

\subsection{Contact Angle Measurements And Surface Tension Determination}

The contact angle was measured by using an OCA 15 plus goniometer (DataPhysics, Filderstadt, Germany) following UNE EN 828:2009. The contact angle was studied in order to evaluate the wettability of the adhesive with different neoprene contents on the two adherends with different levels of roughness $(\mathrm{Ra}=0.2 \mu \mathrm{m}$ and $\mathrm{Ra}=2 \mu \mathrm{m})$. Six adhesive formulations $(8,10,11,11.5,12$ and $12.5 \mathrm{wt} \%$ neoprene) were tested. The determination of surface tension was carried out with the same equipment using the Pendant Drop Method. The adhesive pendant drop dispensed was $3 \mu \mathrm{L}$ and the image was captured after 10 seconds. SCA20 software (DataPhysics, Filderstadt, Germany) was used to determine surface tension. Five adhesive formulations with different contents of neoprene $(8,10$, 12,14 , and 16 wt $\%$ ) were tested.

\subsection{Lap-Shear Strength}

The effect of the adhesive viscosity on the joint strength was evaluated by tensile shear tests using single-lap joints according to UNE EN 1464:2010. The tests were performed in an universal testing machine Ibertest ELIB 20W (Ibertest, Madrid, Spain). The specimens had two rectangular adherends. The dimensions of the adherends were $25 \mathrm{~mm}$ 
wide, $100 \mathrm{~mm}$ long and $1.6 \mathrm{~mm}$ thick. The sections were bonded together with an overlap length of $12.5 \mathrm{~mm}$. The average bondline thickness was $0.8 \pm 0.1 \mathrm{~mm}$ and was cured for 72 hours under controlled temperature $\left(23 \pm 2{ }^{\circ} \mathrm{C}\right)$ and humidity $(30 \pm 5 \%)$. A jig was used to achieve the bondline thickness and to ensure proper alignment [18]. Six adhesive formulations with different contents of neoprene $(8,9,10,12,14$ and 16 wt $\%)$ were tested. The values shown in the graphs correspond to an average of five test specimens and the bars indicate the standard deviations.

\subsection{Scanning Electron Microscopy}

In order to analyze the effect of the viscosity and the roughness on the joint interface, single-lap specimens were prepared and a section was cut according to Figure 1, using a low-speed water-cooled diamond saw (Minitom, Struers A/S, Copenhagen, Denmark). The joint section was observed by scanning electron microscopy (SEM) using a Philips XL-30 microscope (Philips, Eindhoven, Holland). The samples were prepared using gold coating in a high-resolution Polaron SC7610 sputter coater (VG Microtech, Uckfield, United Kingdom) in order to obtain a conductor media for electrons and sufficient contrast in the SEM micrographs. The energy of the electron beam was $20 \mathrm{kV}$.

\section{RESULTS AND DISCUSSION}

In Figure 2 an increase in the neoprene concentration can be observed, this produces an increase in the viscosity and surface tension of the adhesive. The adhesive, before the curing reaction, basically consists in a solution of a polymer (neoprene) on a solvent (acrylic monomers). The polymer dissolution on the solvent involves the disaggregation 
of their macromolecules and the diffusion into the solvent, forming a single phase. The cohesive forces, which initially hold the macromolecules linked, are weakened and the distance between the macromolecules chains is reduced.

The interaction between chains depends on the degree to which the solvent molecules reduce the intermolecular contact, and therefore weaken the cohesive forces between the polymer chains. When the solution has high concentration of neoprene, the disaggregation of the polymer macromolecules is low; in consequence the cohesive forces between their macromolecules are strong [19]. These higher intermolecular forces lead to an increment of viscosity and surface tension.

Figure 3 shows the contact angle of the adhesive as a function of the adhesive viscosity, measured on two adherends with different levels of roughness $(\mathrm{Ra}=0.2 \mu \mathrm{m}, \mathrm{Ra}=2 \mu \mathrm{m})$. As can be observed, an increase in viscosity produces an increase in contact angle for both levels of surface roughness. As discussed before, the increase in the viscosity means an increase in the surface tension of the adhesive and therefore a reduction in wettability [20].

The adhesive samples prepared with up to $10 \%$ neoprene may be considered as low viscosity adhesives, and the adhesives samples with higher concentrations may be considered as high viscosity adhesives. Thereby, when the two levels of roughness are compared, it is possible to divide Figure 3 in two well differentiated parts. In Part 1 (low 
viscosity) the contact angle is smaller for the roughest surface. This is in accordance with the Wenzel equation.

However, when the viscosity rises, a point is reached in which this trend is reversed and the contact angle is smaller in the less rough surface. This is Part 2 of Figure 3 (high viscosity) and in this part, the Wenzel's equation is not satisfied.

Before discussing these results it is important to emphasize that the Wenzel equation assumes two ideal situations: the surface energy of both adherends (smooth and rough) is similar and the penetration of the adhesive into the superficial cavities and roughness is complete $[21,22]$.

However, it is possible that one or both of these ideal assumptions are not satisfied and for a certain value of viscosity, the Wenzel equation might fail. It would be possible to consider two hypotheses about why, beyond a certain degree of viscosity, and depending on the roughness of the adherend, the Wenzel equation becomes invalid.

The first hypothesis considers that the surface treatment not only modifies the topography of the adherend, but also modifies the physicochemical properties (e.g. surface chemistry and surface energy) [16]. These modifications may cause differences in the wettability between the adherends studied, which cannot be attributed exclusively to the differences in the roughness [23]. 
The second hypothesis considers that for relatively high values of viscosity, the penetration into the voids and the copy of the superficial morphology to achieve good wettability may be hampered. This may be especially important in the case of very rough surfaces, or surfaces whose morphology shows very sharp peaks and valleys.

In this study, it has been observed that in the case of low viscosities, the adhesive is capable of copying the surface morphology perfectly. Thus a good penetration of the adhesive into the surface microcavities is achieved, avoiding the formation of voids that would lead to joint failure. Furthermore, the intimate contact adhesive-adherend is improved.

Figure 4 shows the joint interface in the case of a low viscosity adhesive ( $8 \%$ neoprene), the micrographs demonstrate a good wettability: the adhesive (on top) is an exact copy of the morphology of the adherend (on the bottom). The penetration into the microcavities of the surface is complete, not only in the case of substrate with low roughness (Figure 4a), but also in the case of rough substrates (Figure 4-b and Figure 4-c).

However, Figure 5 shows how in the case of adhesive samples with high viscosity, the copy of the morphology of the adherend by the adhesive is not as exact as in the case of low viscosity adhesives, this is a consequence of a poorer wettability and a worse interfacial contact. Considering a good wetting as a good spreading of the adhesive on the surface, this leads to the achievement of a good contact. This phenomenon is observed in 
the SEM images as a true copy of the surface by the adhesive. It is independent of the gap distance between the adhesive and adherend produced by the shrinkage.

Figure 5 -a shows the interface of an adhesive with $10 \%$ neoprene and Figure $5 \mathrm{~b}$ of an adhesive with $12 \%$ neoprene (Figure 5 -b), using the substrate with a high roughness of $2 \mu \mathrm{m}$ in both cases. In the areas marked in these images is possible to appreciate how the adhesive with $10 \%$ of neoprene (low viscosity) is able to copy the morphology of the adherend better than in the case of the adhesive with $12 \%$ of neoprene (high viscosity); as a consequence, the wettability and the intimate contact are more favorable.

This trend is observed even in the case of the low roughness substrate, Figure 5-c and Figure 5-d compare the interface quality for a roughness of $0.2 \mu \mathrm{m}$ in the case of a low viscosity adhesive with $10 \%$ of neoprene (Figure 5-c) and a high viscosity adhesive of $16 \%$ of neoprene (Figure 5-d). The decrease of the wettability and of the contact between adhesive and substrate is considerable with the viscosity rise; this effect is in agreement with the contact angle results reported above.

In relation to the micrographs commented above, it is important to highlight some other effects observed as a consequence of the increase in the viscosity, which could modify the adhesive joint strength.

When Figure $4-\mathrm{a}$ ( $8 \%$ Neoprene), Figure 5 -a (10\% Neoprene) and Figure 5-b (12\% Neoprene) are compared, it is possible to note that the distance that exists in the interface 
between adhesive and substrate is greater in the case of the adhesive with a lower neoprene content, which also means lower viscosity. This is about $4.4 \mu \mathrm{m}$ in the case of the adhesive with $8 \%$ of neoprene, and of $1.8 \mu \mathrm{m}$ and $1.5 \mu \mathrm{m}$ in the case of $10 \%$ and $12 \%$ of neoprene, respectively. The gap observed in the micrographs is a consequence of the adhesive shrinkage that happens during the curing process, which is considerably reduced when the neoprene content of the adhesive is increased. Initially, when the adhesive is applied, it spreads by the surface, wetting the adherend and making a perfect copy of the surface morphology. However, during the curing, the polymerization of the acrylic adhesive can result in a large adhesive shrinkage [24].

Figure 6 shows an example of the quality of the interface, where it is possible to observe that the shrinkage of the adhesive mainly has two consequences on the quality of the joint. On the one hand, the number of observed contact points along the interface is reduced. Also the breakage of the bonds, which were formed before the contraction of the adhesive, is observed. Although initially when the adhesive is applied, a good adhesion could be formed, the shrinkage during the curing process promotes the interfacial failure. In addition, the contact points due to chemical interactions are short range forces, thus they directly depend on the distance between adhesive - adherend, as for example, the Van der Walls forces. On the other hand, this shrinkage may also generate residual stresses that can cause detrimental effects and can damage the joint strength $[25,26]$.

Figure 6 -a corresponds to the adhesive with $16 \%$ of neoprene (high viscosity). In this case, in the area marked with a circle it is possible to appreciate the low contraction of the 
adhesive (short distance adhesive-adherend), consequently, a substantial number of contact points may be observed and the distribution of these along the interface is homogeneous. However, Figure 6-b, which corresponds to the adhesive with $8 \%$ of neoprene (low viscosity), shows that the bonds were broken due to the contraction (see circles in the figure), and the distribution along the interface is less homogeneous that in the other case.

The effects discussed above, which are a consequence of the increase in the neoprene content (viscosity), affect in some way the final adhesive joint strength. Figure 7 represents the variation of the average shear strength with the viscosity of single lap joint specimens. The results for both adherends used in this study are represented. In order to make the discussion of the results easier, the average shear strength graph is divided into two parts.

Part 1, marked in Figure 7 and represented in detail in Figure 8, corresponds to the low values of viscosity. At these values, the observed trend is particularly different to the rest of the graph. The strength is generally low, but is lower in the case of adherend with $R_{a}=2$ $\mu \mathrm{m}$ than in the case of $\mathrm{R}_{\mathrm{a}}=0.2 \mu \mathrm{m}$. In addition, the strength continuously increases in the case of the rough surface with the viscosity, however in the case of the surface with $\mathrm{Ra}=0.2 \mu \mathrm{m}$ it is possible to note a punctual decrease of the strength.

This is mainly explained, on the one hand, by the low toughness of the adhesive when the neoprene content is low. The negative effect of the low toughness is especially 
remarkable in rough adherends. The rough surfaces present more stress concentration points that facilitate the cracks propagation if the adhesive is not tough enough [27]. On the other hand, the breakage of the bonds due to shrinkage is especially important for these low neoprene content adhesives.

In contrast to the previous case, in part 2 of Figure 7, the joint strength is greater in the case of adherends with high roughness; in spite of this, it is important to note that the trend is similar for both adherends used: the average shear strength increases with the viscosity increase until it reaches an optimal point. From this point, for values of higher viscosity, the strength decreases. For very elevated viscosities, the strength decreases considerably.

To explain the observed trend in the strength with the viscosity variation, it is necessary to consider that the viscosity increase produces different effects in the behavior of the adhesive joint. Some of these effects are positive, however, some others may adversely affect the joint. Consequently, as the variation of the viscosity of the adhesive does not affect a single property, it is necessary to consider the overall balance of the change in each of the affected properties, in order to understand the global result on the final strength.

Table 2 summarizes how the increase of adhesive viscosity affects the different properties of the adhesive joint; most of them were discussed above. Also, Table 2 indicates if this 
modification in the adhesive properties may improve the final strength, or if otherwise may negatively affect the joint.

Considering the way in which these properties are affected by the viscosity increase, the results obtained can be discussed by dividing Part 2 (Figure 7) into two segments. On the first segment, the increase in the viscosity enhances the bond strength. This is due to the decrease in the adhesive-adherend distance, improvement in the toughness of the adhesive, reduction of the shrinkage during curing, and the improvement in the quality, strength and uniformity of the chemical bonds. Adhesive penetration into the structure created by the surface treatment is a very important issue to improve the durability of the adhesive joint [28]. Arrowsmith et al. [29] reached a similar conclusion working with aluminum and toughened acrylic adhesives, indicating that to ensure good behavior of the joint, the adhesive must be selected so it can penetrate into the pores of the oxide layer.

On the second segment, viscosity achieves an optimum point in which strength is maximized, and in which a further increase in the viscosity leads to a decrease in the resistance. This is due to the negative effect of the decrease in the wettability and in the homogeneity of the interface of the adhesive. This overrides the positive effects discussed above, and the increase in the viscosity starts to become negative for the strength of the joint. In summary, the viscosity increase leads to less adhesive wettability, especially in the case of adherends with high roughness, and this phenomenon negatively affects the joint strength. 
In contrast, the increase in the viscosity as a consequence of the large neoprene content has some positive effects for the adhesive joint. Some of these positive effects are the reduction in the adhesive shrinkage for high neoprene content adhesives, which in turn cause a lower distance between the adhesive and the adherend. The consequences are stronger bonds due to chemical interactions and less breaking of the contact points formed, resulting in a better quality and quantity of interactions between adhesive and adherend in the interface.

Moreover, the content in neoprene also improves the adhesive toughness, and this may enhance the strength of the joint, especially in the case of rough adherends in which the surface morphology may induce stress concentration points in the adhesive [30].

The balance between positive effects and negative effects causes that, when the viscosity is low, an increase in the viscosity leads to an improvement in the joint strength (because the positive effects are stronger than the negative effects). On the contrary, when the viscosity is high, an increase in the viscosity leads to a decrease in the joint strength, mainly due to the poor wettability.

\section{CONCLUSION}

The results obtained in this study show the relevant role of the adhesive viscosity in the adhesion strength. It has been observed that, regardless of the substrate roughness, the adhesive shows an optimal viscosity for which the joint strength exhibits a maximum. 
The mechanical test showed that below the optimal viscosity, the low neoprene content produces poor bond strength, mainly due to the high shrinkage during curing observed by SEM analysis of the interface adhesive-adherend. The shrinkage has a double effect on the final strength; on the one hand it leads to apparition of residual stresses, which weakens the interfacial strength. On the other hand, it causes separation between the adhesive and adherend, which leads to a poor interfacial contact. This is due to the reduction of the number of contact points along the interface, the breakage of the bonds formed before the contraction, and the weakness of the chemical interactions caused by a higher distance adhesive-adherend.

The reduced toughness of the adhesive with low neoprene content may also contribute to the low strength found in the adhesives with low viscosity. This is especially notable in the case of the rough adherends, due to an increment of stress concentration points that facilitates the crack propagation. In the case of the adhesives with a viscosity above the optimal value, the measurements of contact angle corroborate that the reduction of the joint strength is the result of an important decrease in the wettability. This poor wettability is due to an insufficient spreading of the adhesive on the surface, this leads in a bad penetration of the adhesive into the surface microcavities which promotes the formation of voids in the interface where the contact adhesive-adherend is poor and negatively affects to the interfacial strength. This effect is more pronounced in the case of rough substrates. 
Table 1. Formulation used in the synthesis of the adhesive

\begin{tabular}{|l|l|l|}
\hline \multirow{2}{*}{ Adhesive } & Methyl methacrylate & $83.3 \mathrm{wt} \%$ \\
\cline { 2 - 3 } & Methacrylic acid & $14.7 \mathrm{wt} \%$ \\
\cline { 2 - 3 } & Ethylene dimethacrylate & $2 \mathrm{wt} \%$ \\
\hline Additives & Hydroquinone & $70 \mathrm{ppm}^{(1)}$ \\
\cline { 2 - 3 } & 4-methoxyphenol & $250 \mathrm{ppm}^{(1)}$ \\
\hline Toughness modifier & Neoprene & $\% \mathrm{depending} \mathrm{on} \mathrm{the}^{2}$ \\
\hline Cure initiator & Ciscosity \\
\hline Activator & $0.2 \%{ }^{(2)}$ \\
\hline Loctite 7388 & \\
\hline
\end{tabular}


Table 2. Summary of the effect of the viscosity increase over the different properties of the adhesive and how this change may affect the strength of the joint

\begin{tabular}{|c|c|c|c|}
\hline Joint property & $\begin{array}{l}\text { Modification of the } \\
\text { property when the } \\
\text { viscosity increase }\end{array}$ & $\begin{array}{l}\text { Effect on joint } \\
\text { strength }\end{array}$ & Figure referenced \\
\hline $\begin{array}{l}\text { Distance adhesive- } \\
\text { adherend }\end{array}$ & Decrease & $\begin{array}{l}\text { Improves strength } \\
\text { of the joint }\end{array}$ & $\begin{array}{l}\text { Figure 4-a, Figure } \\
\text { 5-a and Figure 5-b }\end{array}$ \\
\hline $\begin{array}{l}\text { Wettability and } \\
\text { homogeneity of } \\
\text { interface }\end{array}$ & Decrease & $\begin{array}{l}\text { Impairs stre } \\
\text { of the joint }\end{array}$ & \\
\hline $\begin{array}{l}\text { Quantity and strength of } \\
\text { bonds in the interface }\end{array}$ & Increase & $\begin{array}{l}\text { Improves strength } \\
\text { of the joint }\end{array}$ & Figure 6 \\
\hline $\begin{array}{l}\text { Homogeneity of } \\
\text { adhesive layer }\end{array}$ & Decrease & $\begin{array}{l}\text { Impairs strength } \\
\text { of the joint }\end{array}$ & - \\
\hline Adhesive toughness & Increase & $\begin{array}{l}\text { Improves strength } \\
\text { of the joint }\end{array}$ & - \\
\hline Shrinkage & Decrease & $\begin{array}{l}\text { Improves strength } \\
\text { of the joint }\end{array}$ & $\begin{array}{l}\text { Figure 4-a, Figure } \\
\text { 5-a and Figure 5-b }\end{array}$ \\
\hline
\end{tabular}


Figure 1

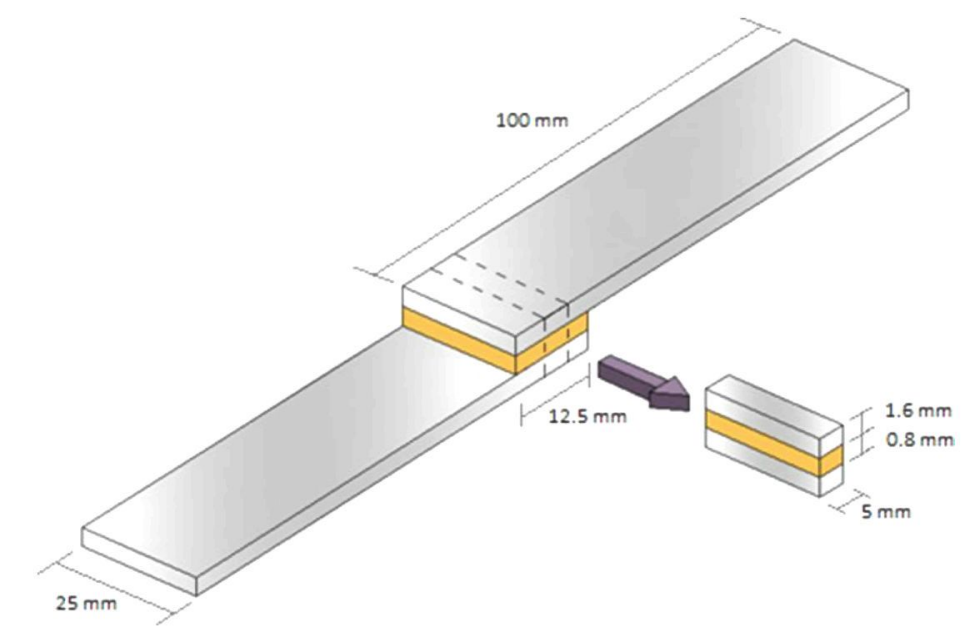


Figure 2

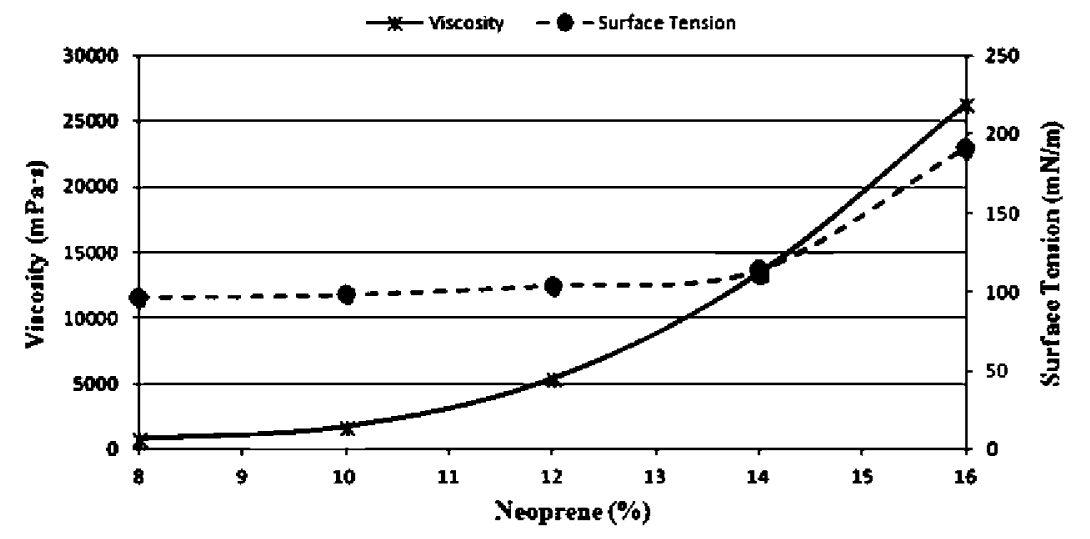


Figure 3

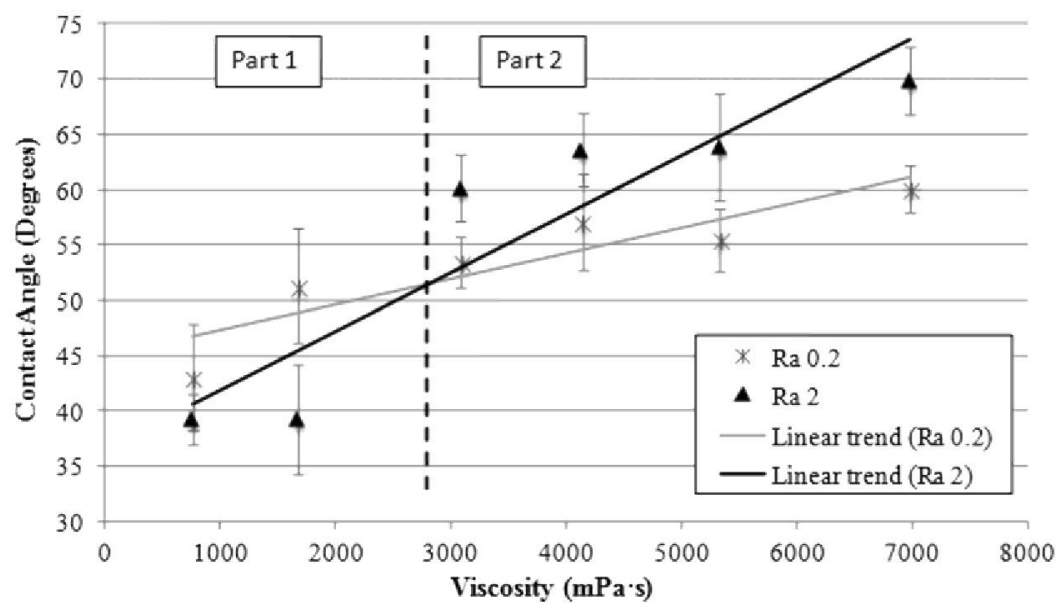


Figure 4

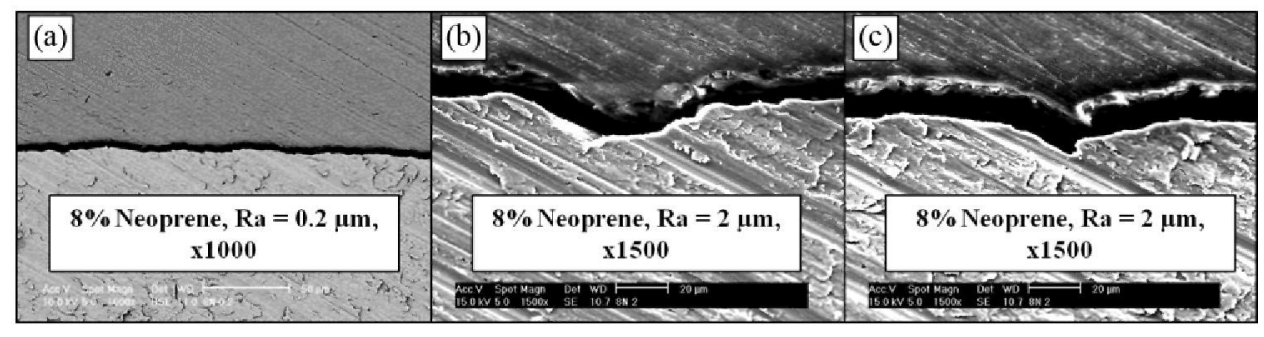


Figure 5

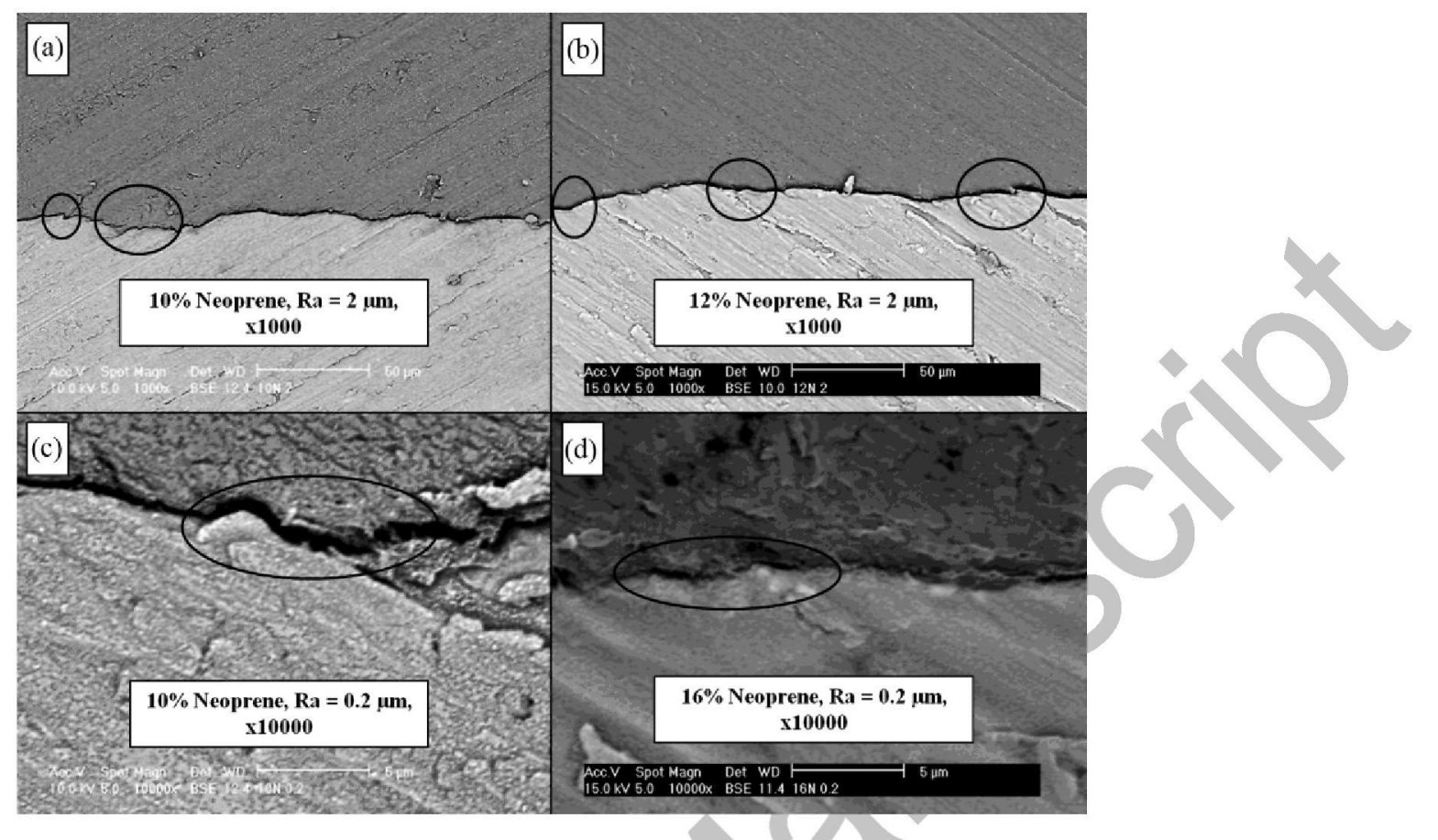


Figure 6

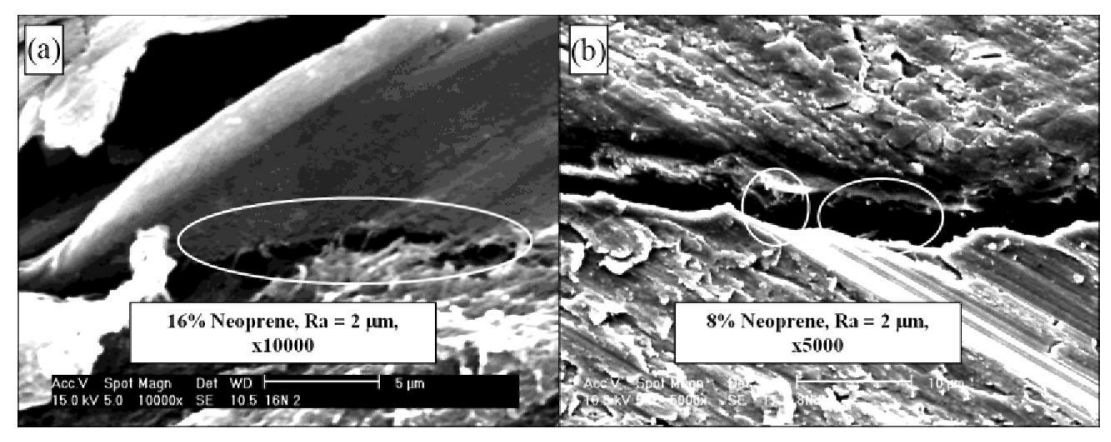


Figure 7

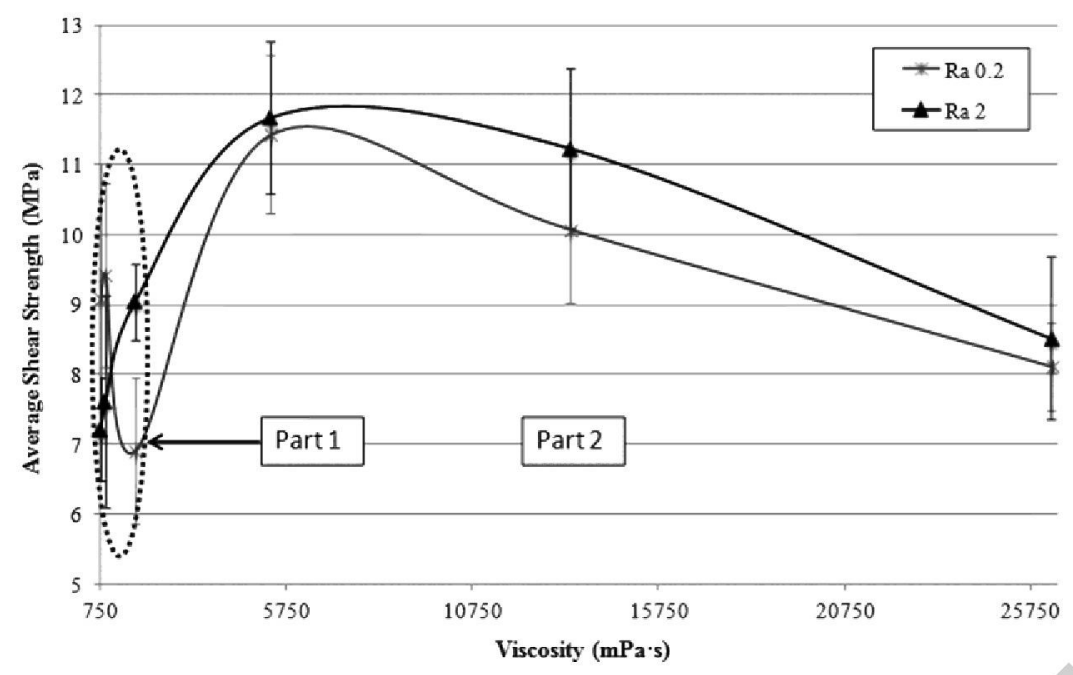


Figure 8

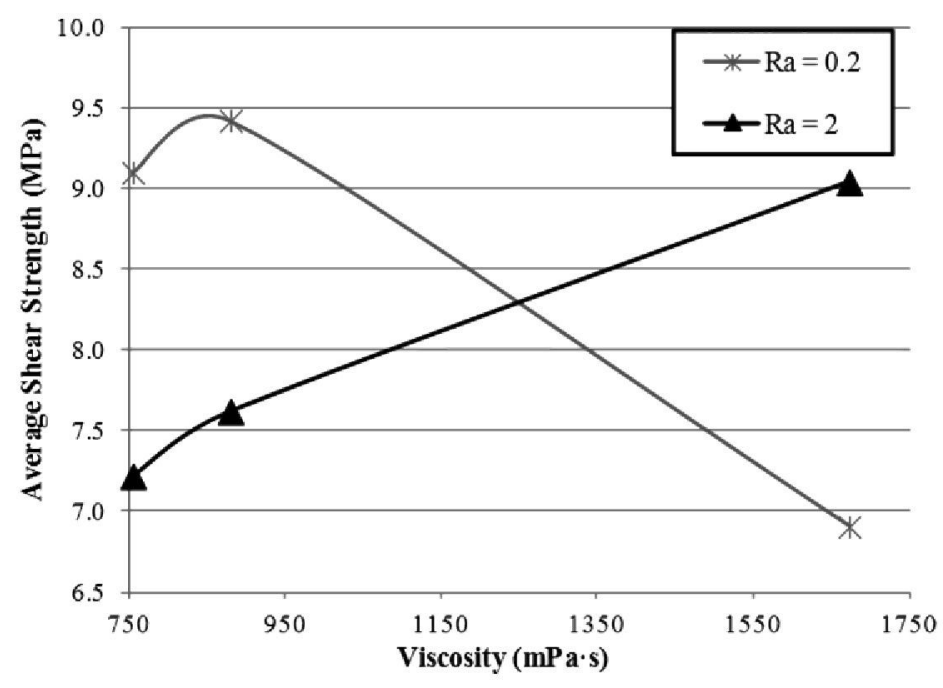

